\title{
THE ELECTRICAL AND THERMAL CONDUCTIVITY OF WOVEN PRISTINE AND INTERCALATED GRAPHITE FIBER-POLYMER COMPOSITES
}

\author{
James R. Gaier*, Yvonne YoderVandenberg**, Steven Berkebile**, \\ Heather Stueben**, and Frederick Balagadde** \\ *NASA John H. Glenn Research Center at Lewis Field, Cleveland, OH 44135 \\ **Manchester College, North Manchester, IN 46962
}

\begin{abstract}
A series of woven fabric laminar composite plates and narrow strips were fabricated from a variety of pitch-based pristine and bromine intercalated graphite fibers in an attempt to determine the influence of the weave on the electrical and thermal conduction. It was found generally that these materials can be treated as if they are homogeneous plates. The rule of mixtures describes the resistivity of the composite fairly well if it is realized that only the component of the fibers normal to the equipotential surface will conduct current. When the composite is narrow with respect to the fiber weave, however, there is a marked angular dependence of the resistance which was well modeled by assuming that the current follows only along the fibers (and not across them in a transverse direction), and that the contact resistance among the fibers in the composite is negligible. The thermal conductivity of composites made from less conductive fibers more closely followed the rule of mixtures than that of the high conductivity fibers, though this is thought to be an artifact of the measurement technique. Electrical and thermal anisotropy could be induced in a particular region of the structure by weaving together high and low conductivity fibers in different directions, though this must be done throughout all of the layers of the structure as interlaminar conduction precludes having only the top layer carry the anisotropy. The anisotropy in the thermal conductivity is considerably less than either that predicted by the rule of mixtures or the electrical resistivity.
\end{abstract}

\section{INTRODUCTION}

To date the high electrical and thermal conductivity of laminar carbon fiber-polymer composites has been largely overlooked when designing aerospace structures. This is in spite of the fact that high-grade graphite fibers have thermal conductivity exceeding that of metals ${ }^{1}$, and when modified by intercalation have electrical conductivity comparable to metals ${ }^{2}$, but at much lower mass densities and higher strengths and modulus. Part of the hesitancy for designers to utilize these materials may stem from the paucity of experimental data on the conductivity properties of these composites. Although there have been many studies of the properties of composites made from chopped fibers in random orientation ${ }^{3}$, and studies of uniaxial continuous fiber composites ${ }^{4}$, there have been relatively few studies done which utilize high conductivity fiber in its most common structural form in the aerospace industry, laminar layups of fiber fabrics ${ }^{5}$.

Investigations carried out by Hambourger in the 1980's indicated that the electrical resistivity of graphite fibers polymer composites which are of a size comparable to the tow size of the fibers had a marked dependence on the geometry of the composite. ${ }^{6}$ Specifically, when the width of the composite dropped below about $4 \mathrm{~mm}$, the resistivity began to increase.

These experiments lead to the question of how the resistance would vary with angle with respect to the weave in such a narrow comnosite. Would these angular effects be visible for composites

This report is a preprint of an article submitted to a journal for publication. Because of changes that may be made before formal publication, this preprint is made available with the understanding that it will not be cited or reproduced without the permission of the 
which are relatively large with respect to the tow size? Further, does the thermal conductivity of the composite follow the same trends as the electrical conductivity? Finally, can the angular dependence of the resistivity be utilized to tailor an anisotropy into the composite? Such channeling of the current could be useful in defining selective area grounding planes or even built-in power buses, and thermal channeling could be used to provide the more cooling to some regions than others. The objectives of this study were to answer these questions.

\section{METHODS AND MATERIALS}

Two classes of composites were fabricated for this study. One was a series of relatively small, narrow composites used to study the angular dependence of the weave. The second class was a series of relatively large composite plates, to study whether angular dependence could be expected to affect the performance of composite space structures.

Narrow composite strips were fabricated under the auspices of two separate NASA Space Act Agreements, one with Applied Sciences, Inc. (Cedarville, $\mathrm{OH}$ ) to intercalate woven carbon fabrics with bromine, and the other with Hughes Space and Communications Company (Los Angeles) to fabricate and test composites for EMI shielding. Two thousand filament tows of Thornel P-100 graphite fiber (BP-Amoco) were woven into a 1 meter wide and several meters long $4 \times 4$ tow $/ \mathrm{cm}\left(10 \times 10\right.$ tow/in) $0-90^{\circ}$ plain weave by Bainbridge Iron and Metal Service (Lyndhurst, $\mathrm{OH}$ ). Half of the fabrics were then intercalated with bromine by Applied Sciences under the guidance of the NASA Glenn Research Center using a vapor fusion technique. The electrical resistivity and thermal conductivity of the fibers used in this study are summarized in Table I. These fibers were preimpregnated with a polyisocyante resin, RS-3, by YLA, Inc. (Benecia, CA). Four-ply, $0-90^{\circ}$ laminar composites approximately $80 \mathrm{~cm}$ square were fabricated by Hughes.

\section{Table I - Properties of fibers used in this study.}

\begin{tabular}{|c|c|c|c|c|}
\hline \multirow[t]{2}{*}{ Fiber } & \multicolumn{2}{|c|}{ Pristine } & \multicolumn{2}{|c|}{$\mathrm{Br}$ Intercalated } \\
\hline & $\begin{array}{c}\rho \\
\mu \Omega-\mathrm{cm}\end{array}$ & $\begin{array}{c}\kappa \\
W / m-k\end{array}$ & $\begin{array}{c}\rho \\
\mu \Omega-\mathrm{cm}\end{array}$ & $\begin{array}{c}K^{*} \\
W / m-K\end{array}$ \\
\hline$T-300$ & 1800 & 8.5 & & \\
\hline P-55 & 850 & 100 & 300 & 90 \\
\hline P-75 & 750 & 155 & 280 & 140 \\
\hline P-100 & 250 & 520 & 50 & 475 \\
\hline
\end{tabular}

Composite strips $9 \mathrm{~mm}$ wide and $100 \mathrm{~mm}$ long were cut from this composite using a band saw. This width was chosen so that each sample would have more than a single tow in each direction. Samples were cut with the axial direction being $0^{\circ}, 15^{\circ}, 30^{\circ}$, and $45^{\circ}$ with respect to the weave direction. Current leads were formed by wrapping fine copper wire around each end, and then applying silver paint (SPI, West Chester, PA) over the wires and over the ends of the composite. 
This was to ensure that the current (via a Keithley Model 225 Constant Current Source) was applied evenly into the sample. Silver paint voltage contacts about $3 \mathrm{~mm}$ wide were applied to the cut edges of the composites at $8 \mathrm{~mm}$ intervals. Contact to a Keithley Model 181 Nanovoltmeter was made through a series of spring-loaded copper contacts of similar spacing. Voltage measurements were recorded for all pair-wise combinations of voltage contacts at both polarities and averaged to negate electrochemically induced voltages. Using the least-squares plot of resistance as a function of the ratio of the sample length to the cross-sectional area greatly enhanced the accuracy of the resistivity of the narrow composites. A typical example of such a plot is shown in figure 1. It is noted that pair-wise measurements would have given slopes (resistivities) over a fairly broad range of values.

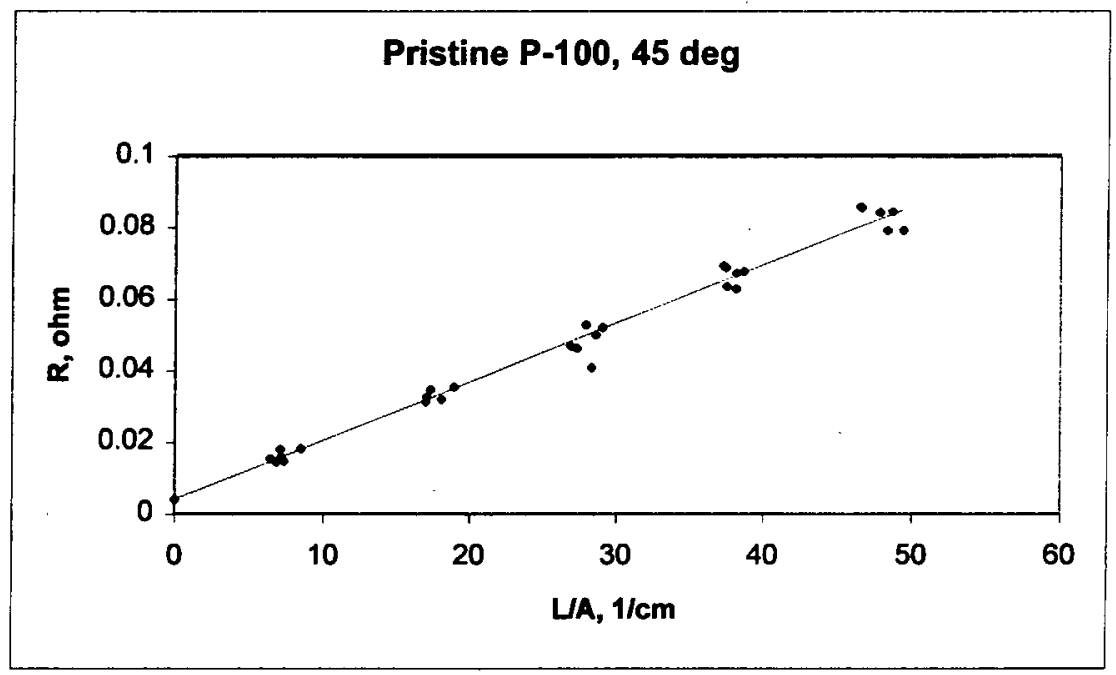

Figure 1 - Typical plot of the resistance as a function of the ratio of the distance between points to the cross sectional area. The slope of such a plot yields the resistivity of the sample.

In addition, several $5 \mathrm{~cm}^{2}$ samples were cut from this composite. Grid lines were marked at 6.5 $\mathrm{mm}$ intervals both horizontally and vertically, and the thickness and conductivity were determined at the intersections using a digital micrometer. The electrical conductivity was measured at each of these intersections by the contactless eddy-current technique using a Leheighton Electronics Model LEI 1010A which was factory modified to operate at $55.55 \mathrm{kHz}$. . At this frequency, the skin depth varies from 3 to $6 \mathrm{~mm}$ depending on the resistivity of the composites, but well beyond the $1 \mathrm{~mm}$ thickness of the composites for the resistivity measured Only the points judged not to include edge effects were used in the analysis.

Larger composites plates were fabricated at a later date from a variety of three grades pitchbased graphite fibers, Thornel P-55, P-75, and P-100, and PAN-based T300. Six thousand filament tows of P-55 and P-75 fibers were woven into a $4.3 \times 4.3$ tow $/ \mathrm{cm}(11 \times 11$ tow $/ \mathrm{in}) 0-90^{\circ}$ five harness satin weave by Mutual industries (Philadelphia, PA). Two-thousand filament tows of P-100 were woven in a $0-90^{\circ}$ four harness satin weave, and were used as the weft with a fabric with T-300 warp by Fabric Development (Quakertown, PA). Samples of each of these fabrics were intercalated with bromine. Bromine intercalation was carried out at room temperature, except for the P-55 fibers which were intercalated at $0^{\circ} \mathrm{C}$, in the vapor phase. Synthesis details are available elsewhere ${ }^{5}$. A summary of the fiber properties is found in Table I. The T-300 fibers were essentially unaffected by the bromine treatment ${ }^{~}$. Four-ply, alternating 0 - 
$90^{\circ}$ laminar composites using each of the fiber types, pristine and bromine intercalated, were fabricated with RS-3 resin by YLA. In addition 1, 2, and 6 ply composites of P-100 fabrics were fabricated, as were $0-0-0-0^{\circ}$ composites of the T-300/P100. Each of the composites was $28 \mathrm{~cm}$ square and approximately $1 \mathrm{~mm}$ thick. The thickness of each composite was accurately determined over my measuring it with a digital micrometer $2.5 \mathrm{~cm}$ from the perimeter, and intervals of $2.5 \mathrm{~cm}$. A $28 \mathrm{~cm}$ square, $0.86 \mathrm{~mm}$ thick painted brass sheet was also fabricated as a reference material.

Eddy current composite resistivities were measured at about $3 \mathrm{~cm}$ in from each of the four corners of the $28 \mathrm{~cm}^{2}$ samples using the modified Leheighton 1010A. Samples were rotated from $0^{\circ}-90^{\circ}$ in $15^{\circ}$ increments to detect any angular dependence of the eddy current measurement.

Four-point measurements were made on each of the large composites plates using a constant current of 1.000 A provided by a Keithley Model 228A Current/Voltage Source. The current input contacts were made by first painting across the corner of the composite with silver paint which covered both sides and the edge. A piece of copper foil was placed over this to minimize scratching of the paint, and to this a spring-loaded clamp to which the current lead was attached. A series of similar current output contacts was painted at each of the other three corners, and along the sides at locations to make $15^{\circ}$ increments with the weave direction.

The voltage drops were measured using a Keithley Model 181 nanovoltmeter. A silver paint reference arc was painted 2-3 mm from the current input electrode to force an equipotential and capture the entire current. A $5 \mathrm{~cm} \times 5 \mathrm{~cm}$ grid of silver contacts was painted on the surface of the composite so that equipotential maps could be generated. A plexiglass sample holder supported the composite sheet and aligned spring-loaded voltage contacts in a reproducible way. In some cases higher resolution was obtained by painting contacts on a one $\mathrm{cm}$ grid over some areas of the composite. The surfaces or edges of the composites were not sanded or treated in any way to enhance the contact.

Thickness varied somewhat throughout the fibers and so the thickness was measured at each point where the resistance was measured. The measured thickness was used to determine the resistivity.

Thermal measurements were made using two different heating techniques.. In the first, the samples were resistively heated by applying $10-53 \mathrm{~A} \mathrm{dc}$, and measuring the temperature across the face of the composite with an Inframetrics Model 600 IR Scanner. This instrument is equipped to measure isothermal contours. The data were recorded for later analysis on a VCR. Although this technique allowed us to visualize the pattern of heat flow through the samples, the inability to quantify the applied power limited its ability to quantify the thermal conductivity.

In an attempt to better quantify the thermal conductivity a second technique was used in which the composites were heated using the collimated beam from a quartz tungsten halogen lamp (Oriel Model 6618) which provided up to 1.2 watts of radiant power. The data were recorded using the same IR scanner and analysis techniques described above. Measurements were carried out both in air, and in a $10^{-4} \mathrm{~Pa}$ vacuum. This was a steady state measurement measured at three different radiant power levels. Thermal conductivity $(\kappa)$ was extracted from these data by measuring the circular thermal gradient generated this way using: 


$$
\kappa=\frac{Q}{\left[\frac{2 \pi L\left(T_{2}-T_{1}\right)}{\ln \left(r_{2} / r_{1}\right)}\right]}
$$

where the $L$ is the sample thickness, $T_{n}$ is the temperature measured at radius $r_{n}$ from the center of the spot where the heat was applied, and $Q$ is the magnitude of the heat applied to the sample which was determined optically from:

$$
Q=P_{\text {meas }}\left(\frac{A_{\text {sample }}}{A_{\text {det ected }}}\right)
$$

$P_{\text {meas }}$ is the total power read from the power meter, $A_{\text {sample }}$ is the absorbance integrated over the wavelengths from 300 to $2500 \mathrm{~nm}$ of the sample as determined by the spectrum measured on a Perkin Elmer Model Lambda 21 spectrophotometer with an integrating sphere, and $\mathrm{A}_{\text {detected }}$ is the absorbance integrated over all wavelengths of the detector $(425-1100 \mathrm{~nm})$, as determined by the calibration spectrum provided by the manufacturer.

\section{RESULTS AND DISCUSSION}

The resistivity measured on the narrow composite strips revealed a dependence on the angle of measurement with respect to the weave direction. Sample reproducibility was very good. The angular dependence on the resistivity fit a very simple model. The first assumption of the model is that the current travels only along the axis of the fibers, that is, that the transverse resistivity of the fibers is much higher than the axial resistivity. This is supported by measurements in metal matrix composites which indicate that the transverse resistivity of the fiber is about $10^{2}$ higher than the axial resistivity. ${ }^{8}$ The current can only transverse one fiber diameter, and then it must travel through the resin, which has a very high resistivity $\left(>10^{13} \Omega-\mathrm{cm}\right)$. The second assumption is that the contact resistance between fibers within the weave is negligible. This is plausible because each axial fiber crosses several thousand transverse fibers, in effect a parallel circuit of several thousand resistors. In effect, this model is the rule of mixtures, but taking into account that the fibers are not randomly oriented. Only those fibers normal to the equipotential surface will carry current. For example, in the case where the current travels in the $\theta=0^{\circ}$ direction, the fibers in the $\theta=90^{\circ}$ carry no current, so the effective fiber fill is only one-half of the actual fiber fill.

Thus, the resistance along a path with angle $\theta$ with respect to the fibers would be increased by its path length, leading to $\rho_{\theta}=\rho_{\text {fiber }}(|\cos \theta|+|\sin \theta|)$. Figure 2 shows a polar plot of the data for the $\mathrm{Br}_{2}$ intercalated $\mathrm{P}-100$ composite along with the model predictions. A similar plot was generated for the pristine P-100 composite data. The model predicts the experimental data within 2 percent. 


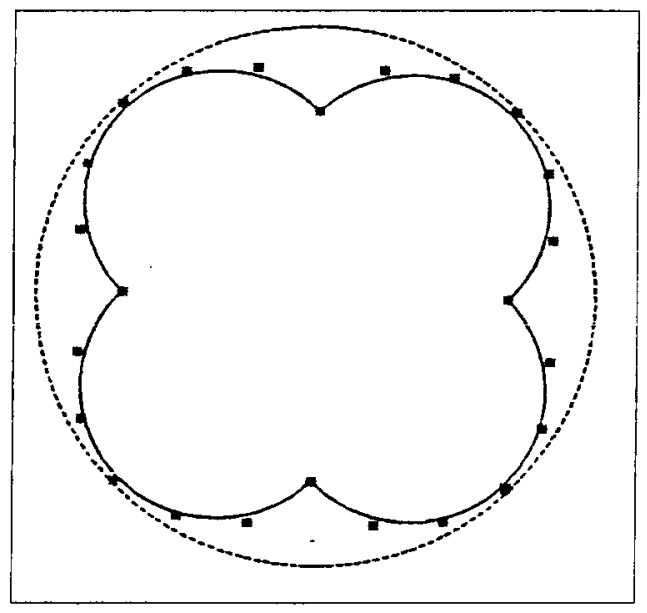

Figure 2 - Polar plot of the angular distribution of the resistivity as a function of angle from weave direction showing the model (solid line), the experimental multipoint values (*), and the eddy current resistivity (dotted line).

The eddy current measurement instrument was built according to the principles put forward by Miller, et al. ${ }^{9}$ It is, in essence, an ammeter measuring the power dissipated by eddy currents induced in the sample. The eddy currents, however, are assumed to be generated in an isotropic medium, which these composites obviously were not. The resistivities of the $5 \mathrm{~cm} \times 5 \mathrm{~cm}$ composites cut from the sample samples as the composite strips were measured to be higher than those measured using the multi-point method, implying that is takes more energy to generate eddy currents in anisotropic media. It was noted, however, that the resistivity measured using the eddy current method was similar to that measured on the $45^{\circ}$ samples using the multipoint method. Since the eddy current resistivity of the coupons was found not to be dependent on the orientation, it is shown as a dashed circle on the polar plot in Figure 2. It is possible that the value is fortuitous, or it may help reveal the natures of eddy current generation in anisotropic media.

Measurements on the large composite plates were carried out to see whether there was anisotropy induced in larger structures by the fiber weave. Equipotential contours were plotted for each of the composite plates using the data from the four-point measurements for each of the current orientations. Two representative samples of the homogeneous composites (i.e. warp fiber same as weft fiber) are shown in Figure 3. These equipotential contours show that, on a cm scale, these composites act as homogeneous plates. To confirm this, a brass plate of identical dimensions was painted and measured using the same apparatus and techniques. The contour diagrams of the brass plate and all of the isotropic composite plates were the same, except for the constant factor of resistivity.

Analysis of these contours enabled the determination of the resistivity of each of the composites. Composite resistivity was calculated for the isotropic composites by examining the voltage gradient when the current leads were at opposite corners of the sample. These measured resistances were fit by the equation: 

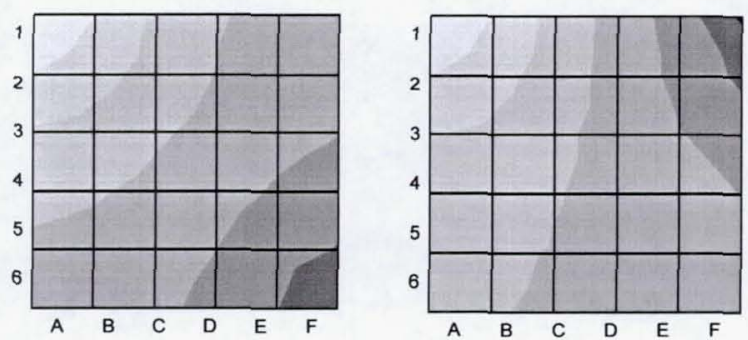

Figure 3 - Equipotential contours on $0-90^{\circ} \mathrm{P}$ 100/RS3 composites with current injected through opposite corners, $1 \mathrm{~A}$ to $6 \mathrm{~F}$ (left) and through adjacent corners, $1 \mathrm{~A}$ to $1 \mathrm{~F}$ (right).

$$
R=\rho\left(\int_{0}^{L_{\text {ref }}} \frac{d L}{\left(C_{\text {ref }}+L_{\text {ref }}\right) t}+\int_{0}^{L_{\text {meas }}} \frac{d L}{\left(C_{\text {meas }}+L_{\text {meas }}\right) t}\right)
$$

where $\mathrm{C}_{\text {ref }}$ and $\mathrm{C}_{\text {meas }}$ are the distances from the near corner of the sample to the reference electrode and the distance from the far corner to the measurement electrode respectively and $\mathrm{L}_{\mathrm{ref}}$ and $\mathrm{L}_{\text {meas }}$ are the distances from the reference electrode to the diagonal and the measurement electrode to the diagonal respectively, and $\mathrm{t}$ is the average thickness. In this analysis the resistivity $(\rho)$ is treated as an empirical factor found by a least squares fit to the data. There was excellent agreement between the model and the data as can be seen in the typical example shown in Figure 4.

\section{P100, 45 degree Resistance}

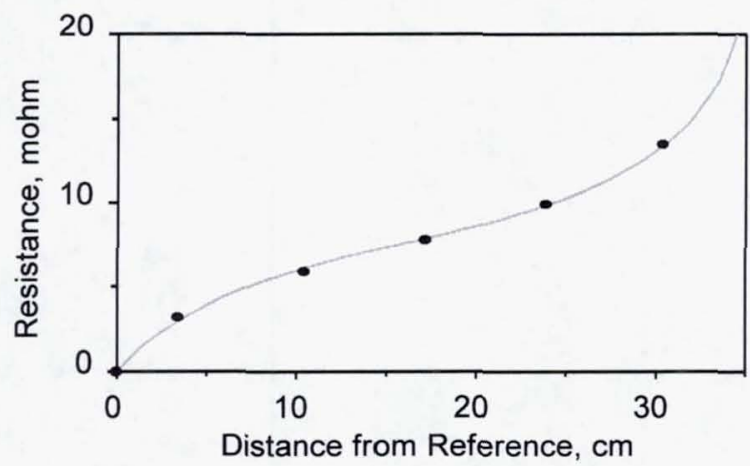

Figure 4 - Fit of the measured data points $(\bullet)$ to the isotropic model described in the text (line).

These values, along with those measured using the eddy current method, and those predicted by the rule of mixtures modified as described above for the $45^{\circ}$ from the weave case are shown in Figure 5. Note that there is agreement between the two experimental methods about the order of 
composites from least to most conductive, but that the eddy current methods gives a value for the resistivity that is about 50 percent higher than the multi-point method. This is surprising given the results from the narrow composites, illustrated in Figure 2, in which the eddy current resistivity values were within a few percent of the values where the current was applied at $45^{\circ}$ with respect to the weave. The modified rule of mixtures predicts the resistivity as measured by the multipoint method generally within 20 percent.

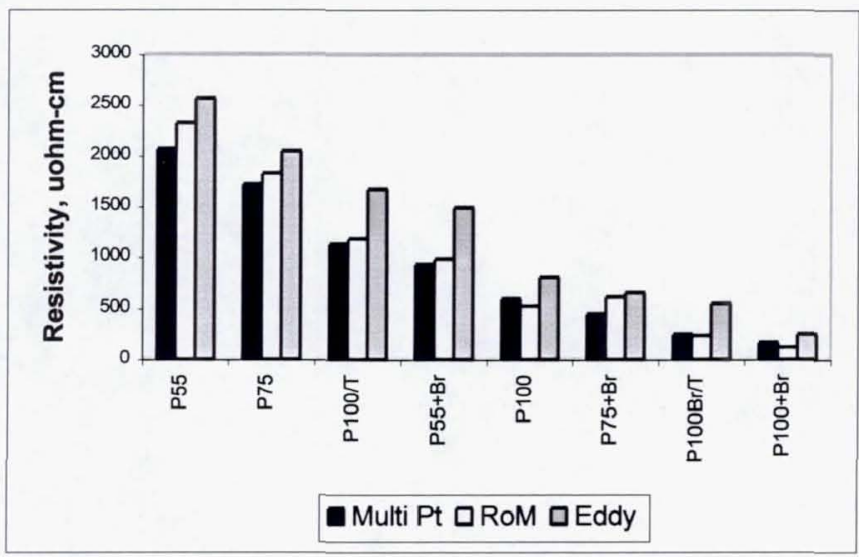

Figure $\mathbf{5}$ - Resistivity of composites as measured by the multi-point and eddy current techniques, and calculated according to the rule of mixtures.

Equipotential contours for the inhomogeneous composites (T-300 warp and P-100 weft) for $0^{\circ}$ $0^{\circ}$ and $0^{\circ}-90^{\circ}$ composites are shown in Figure 6. Note that the $0^{\circ}-90^{\circ}$ contour pattern is virtually identical to those in figure 1 , in contrast to the strong asymmetry in the $0^{\circ}-0^{\circ}$ pattern. This indicates that the layer-to-layer resistance within the laminate is low enough that the top layer cannot dominate the resistance. The veracity of this observation is demonstrated in Figure 5, where it can be seen that the resistivity of $0^{\circ}-90^{\circ} \mathrm{P}-100 / \mathrm{T}-300$ composites, pristine and intercalated, (the third and seventh data sets, respectively) as fit through the $45^{\circ}$ to the weave data is well predicted by the modified rule of mixtures. Thus, if an asymmetric pattern in the resistance is desired, then that pattern must be reinforced through all of the layers.
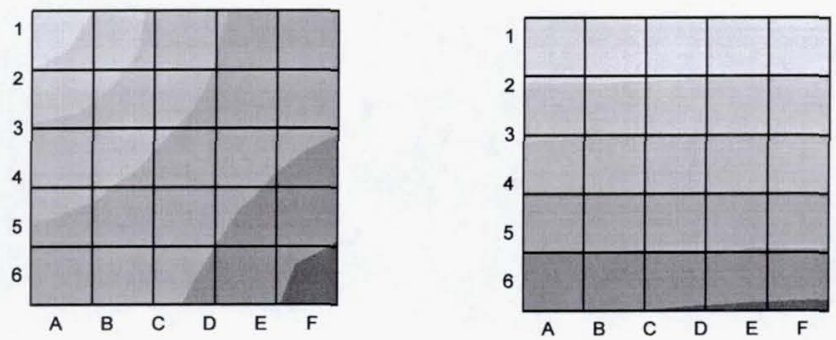

Figure 6 - Equipotential contours on $0^{\circ}-90^{\circ} \mathrm{T}-300 / \mathrm{P}-$ 100 (left) and $0^{\circ}-0^{\circ} \mathrm{T}-300 / \mathrm{P}-100$ (right) composites with current injected through opposite corners, $1 \mathrm{~A}$ to $6 \mathrm{~F}$.

The thermal conductivity of the homogeneous composites was determined from the radiant heating of the composites. Those values are shown in Figure 7. The RoM predictions are verified by experiment when the thermal conductivities are relatively low, but far off when the RoM values were very high. At those values the temperature gradient is very shallow, and the 
error in the measurement increases dramatically. The resistance heating method showed these $\kappa$ values to be far too low.

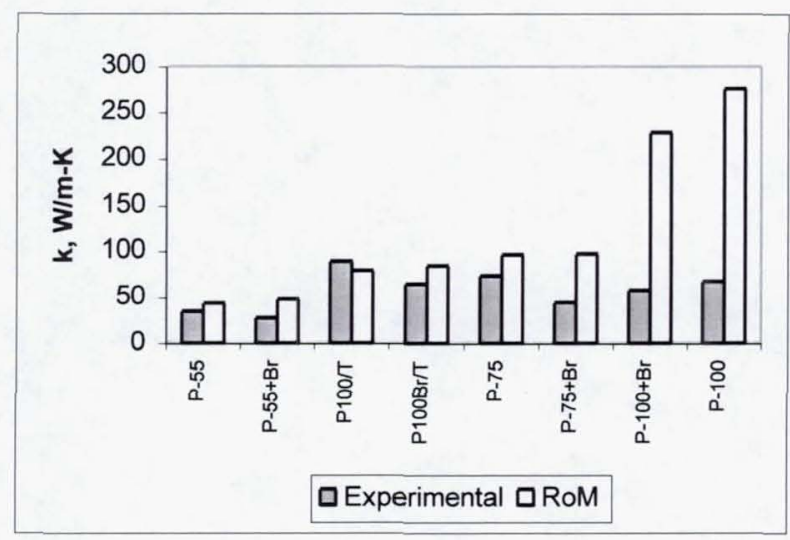

Figure 7 - Experimental and rule of mixtures thermal conductivity on $0-90^{\circ} \mathrm{P}-100 / \mathrm{RS} 3$ composites.

The thermal conductivity measured in the T-300 fiber direction is compared with that in the P100 fiber direction in Figure 8. The RoM predicts the ratio $\kappa$ in the P-100 direction to that in the transverse direction to be 34 for pristine, and 29 for intercalated fibers, but the measured values were 1.8 and 1.1 respectively. This gives additional confirmation that there is appreciable plyto-ply communication, and that this communication is in fact enhanced by bromine intercalation.

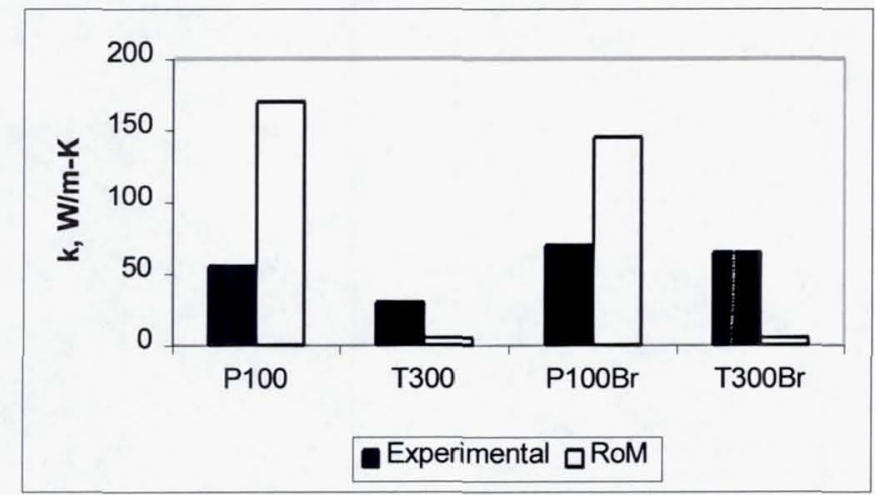

Figure 8 - Experimental and rule of mixtures thermal conductivity of anisotropic composites in the P-100 and $\mathrm{T}-300$ directions, pristine and $\mathrm{Br}$ intercalated $\mathrm{P}-100$.

\section{CONCLUSIONS}

Comparison of the experiments on the composite strips with those of the composite plates gives some guidance as to how these materials can be engineered when electrical properties is a prime consideration. Generally, these materials can be treated as if they are homogeneous plates, much the same as when engineering with homogeneous materials. The rule of mixtures describes the resistivity of the composite fairly well if it is realized that only that component of the fibers normal to the equipotential surface will conduct current. When the composite is narrow with 
respect to the fiber weave, however, there is a marked angular dependence of the resistance which was well modeled by assuming that the current follows only along the fibers (and not across them in a transverse direction), and that the contact resistance among the fibers in the composite is negligible. This results in an effective lengthening of the resistive path length if the fiber weave does not parallel the direction of the potential drop in this region. Thus, if a functional part contains thin strips, or if holes are cut that leave thin strips, then care must be taken that the weave direction does not adversely affect the electrical properties in that region.

Qualitatively, the thermal conductivity results mirrored the electrical resistivity. Quantitatively, the thermal conductivity of composites made from the most highly conductive fibers was much lower than that predicted by the rule of mixtures. This is thought to be an artifact of the measurement technique employed.

If the electrical or thermal anisotropy is desired in a particular region of the structure, it can be achieved by weaving together high and low conductivity fibers in different directions. This must be done throughout all of the layers of the structure, as interlaminar conduction precludes having only the top layer carry the anisotropy. The anisotropy in the thermal conductivity is considerably less than either that predicted by the rule of mixtures or the electrical resistivity. This implies either that the ratio of the transverse to axial thermal conductivity of the fibers is greater than that of the electrical conductivity, or that the thermal conductivity of the resin compared to the fibers is greater than that of the electrical conductivity. Perhaps both are true.

The eddy current technique somewhat overestimates the resistivity of the composites. This may be due to the eddy current size being comparable to the fiber size. The high resistivity would lead the eddy current to be constrained to the fiber, increasing its effective resistance. It is unclear what practical consequences this higher effective resistance may have in the electrical performance of these composites.

\section{ACKNOWLEDGEMENTS}

Y.Y.V, S.B., H.S. and F.B. gratefully acknowledge the support of this work through NASA Cooperative Agreements NCC3-317 and the Ohio Aerospace Institute. The authors would also like to thank G. Clark of Manchester College for many helpful discussions.

\section{REFERENCES}

1. L. Piraux, et al., Solid State Commun. 50 (1984) 697.

2. M.S. Dresselhaus and G. Dresselhaus, Adv. Phy. 30 (1981) 139.

3. F. Carmona, P. Prudhon, and F. Barreau, Solid State Commun 51 (1984) 255.

4. J.C. Abry, et al., Composites Sci and Tech 59 (1999) 925.

5. J.R. Gaier, P.D. Hambourger, and M.E. Slabe, Carbon 29(3) (1991) 313.

6. Private communications, P.D. Hambourger, Cleveland State University (1988).

7. D.A. Jaworske and C. Maciag, NASA Technical Memrandum 100248 (1987).

8. D.L. McDaniels, K.W. Baker, and D.L. Ellis, Proc. $8^{\text {th }}$ Symp. Space Nuclear Power Systems (1991) 313.

9. G.L. Miller, D.A.H. Robinson, and J.D. Wiley, Rev. Sci. Instrum. 47(7) (1976) 799.

10. C-C. Hung and J. Miller, NASA Technical Memorandum 88863 (1986). 